\title{
Boundary Localization of Non-ideal Iris Images Based on Nonlinear Gray-Level Transformation *
}

\author{
Haitao Yu ${ }^{1}$, Jimin Yang ${ }^{1}$, Honglin Wan ${ }^{1,2}$ \\ ${ }^{1}$ Shandong Normal University, No.88 EastWenHua Road, Ji’nan Shandong, China \\ ${ }^{2}$ Southeast University, No.2 Southeast University Road, Nanjing Jiangsu, China \\ jmyang@sdnu.edu.cn, visage1979@sdu.edu.cn
}

\begin{abstract}
Boundary localization is one of the key issues in iris recognition system. For non-ideal iris images, some frequentlyoccurred cases such as dominant texture patterns, eyelashes or eyelids occlusions, low contrast between iris and sclera, and pupil deviation, will lead to inaccurate boundary localization. Specifically, if the intensive transition from iris to sclera is too smooth, outer boundary localization will be very difficult. To stress the problem, in this paper we propose the boundary localization method in which nonlinear gray-level transformation is innovated in outer boundary localization process. The experimental results depict that our algorithm have improved the localization accuracy for non-ideal iris compared to the classical algorithms
\end{abstract}

Index Terms - iris identification, biometrics, iris image preprocessing, iris localization, nonlinear gray-level transformation

\section{Introduction}

With the rapid development of information technologies, personal identification is becoming more and more important. As a result, biometrics technologies have been applied widely. Among the current biometric techniques, iris recognition is regarded as one of the most reliable ones due to its uniqueness, stability and non-invasiveness. Therefore in the recent decade, the research on iris recognition is very active.

A typical iris identification system primarily comprises of image acquisition, image preprocessing, feature extraction (encoding) and feature matching. Particularly, boundary localization in preprocessing step is critical for recognition performance. The first and most well-known boundary localization algorithm is proposed by Daugman [1,2] based on circle-detection due to the normal shape of iris. Another classical approach is presented by Wildes [3] in which edge detection is followed by Hough transform as well as the leastsquares fitting method of Tan et al [4]. During the recent years, boundary localization for non-ideal iris images is a challenge in biometric community. Many researchers have investigated this problem. Roy et al [5] proposed iris segmentation using variational level set method. Pundlik et al [6] introduce the segmentation method by using graph cuts. Miguel et al [7] employ morphology to segment uncalibrated noisy iris data robustly.

There are many degraded cases in iris images such as texture with strong intensive variations, bright spots, eyelashes or eyelids occlusions, low contrast between iris and sclera, and pupil deviation, which perhaps leads to false recognition. In this paper, we propose the boundary localization method based on nonlinear gray-level transformation for non-ideal iris. In inner boundary localization, we locate the coarse position of the pupil by using expectation maximization (EM) segmentation and morphology operation. In resulted pupil region, multiple strings equilibrium approach is introduced to detect inner boundary. In outer boundary localization, the distance-based image enhancement is innovated to strengthen the contrast of sclera and iris region. Then we use Canny detector and circle fitting to locate outer boundary

\section{Proposed Method}

Our proposal includes two phases and the entire flowchart can be seen in Fig.1.

\subsection{Inner Boundary Localization}

\subsubsection{EM based pupil segmentation}

In this step, an iris image is filtered by Laplacian pyramid (LP) [8] to obtain its large scale information. By the means image size will be reduces exponentially. By our observation, it is reasonable that coarse segmentation in the large scale can be refined in small scale. In this idea we decompose iris image $I$ into coarse level firstly. Resulted image $I_{c}$ is segmented by EM algorithm [9] and filtered by morphological close operator to obtain pupil candidate denoted as $I_{c_{-}}$pupil. Iris center is initialized inside it which is optimized by the multiple strings equilibrium. The process described in this subsection is demonstrated in Fig.2.

Because there are some reflective spots in the pupil, we adopt circle structure element denoted by $b$ as the morphological structural element [10] to fill the pupil $I_{c_{-}}$pupil . The process is given as

$$
I_{-} r=\left(I_{c_{-}} \text {pupil } \oplus b\right) \ominus b
$$

where $\oplus$ and $\ominus$ are dilation and erosion operator, respectively.

\footnotetext{
* This paper is supported by Scientific and Technological Development Program of Shandong Province (2011GGB1053), Ji'nan City University Independent Innovation Program (201202018, 201202001), Postdoctoral fund of Southeast University (1109000157).
} 


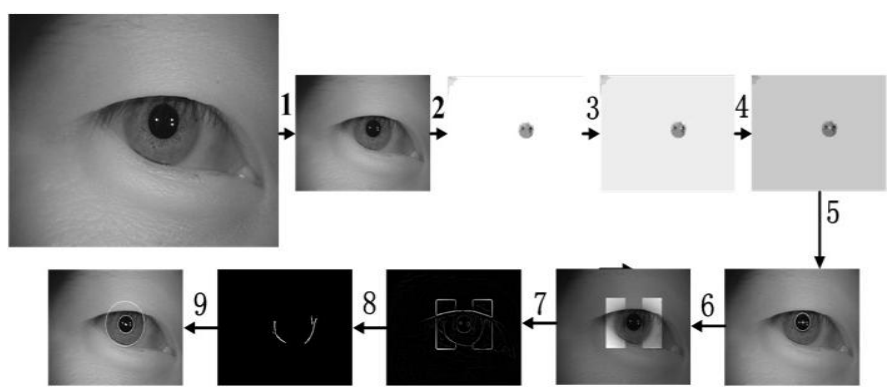

Fig. 1 Flowchart of our method

1. Laplace pyramid decomposition; 2.EM segmentation and close operation; 3. initialization of iris center; 4. optimization of iris center; 5. localization of inner boundary; 6 . nonlinear gray-level transformation; 7. Canny detection; 8 . non-maximum suppression to remove pseudo boundaries; 9.localization of outer boundary by the improved Hough transform.

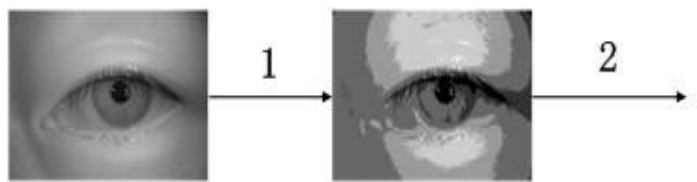

Fig. 2 EM based segment for iris image. 1. Expectation Maximization segmentation, 2. Morphology algorithm removing the bright spots

\subsubsection{The Initialization of the Iris Inner Boundary Center}

To locate the inner boundary accurately, iris center should be initialized inside the pupil. We choose centroid of pupil $I_{-} r$ as the initialized inner boundary center $\left(C_{x}, C_{y}\right)$, i.e.

$$
O=\left(C_{x}, C_{y}\right)=\left(\frac{1}{N_{p u p i l}} \sum_{I_{-}} x, \frac{1}{N_{p u p i l}} \sum_{I_{-}} \sum_{r(x, y) \in L} y\right)
$$

where $N_{\text {pupil }}$ is the number of pixels in the pupil, and L denotes the zero-valued area in the image $I_{-} r$.

\subsubsection{Center Detection and Inner Boundary Localization}

After the initialization of the iris center, three rays are cast from the center $O$ outwards inner boundary in the directions of $0, \pi$ and $7 \pi / 4$, respectively. Considering that upper part of inner boundary is often occluded by eyelashes or upper eyelid, we select the three directions to search inner boundary. Specifically, the three intersections of the rays with candidate pupil region are denoted as $R, L$ and $M$, respectively. We let $\left|D_{1}\right|=|O R|,\left|D_{2}\right|=|O M|,\left|D_{3}\right|=|O L|$. The center $O$ is moved horizontally and vertically until

$$
\left|D_{1}\right|=\left|D_{2}\right|=\left|D_{3}\right|
$$

The radius of the pupil is

$$
R=\left(\frac{\left|D_{1}\right|+\left|D_{2}\right|+\left|D_{3}\right|}{3}\right)
$$

Thereby we localize the iris inner boundary with the parameters $\left(C_{x}, C_{y}, R\right)$.

\subsection{Outer Boundary Localization}

\subsubsection{Gray-level Transformation of the Non-ideal Iris Image}

In this stage, we firstly transform iris image $I_{c}$ into polar domain. The resulted $I \_T$ is processes by the following steps:

1. According to the acquired the center and radius of the inner boundary, we select two symmetrical rectangular areas, $\operatorname{Re} c_{l}$ and $\operatorname{Re} c_{r}$, on left and right sides of the pupil, in either of which there is a part of outer boundary ;

2. Searching the maximum and minimum of gray values of the selected areas, denoted as $I_{\max }$ and $I_{\min }$, respectively;

3. Perform gray-level transform to the selected area by the following formula

$$
I_{-} T(x, y)=\frac{I_{c}(x, y)-I_{\min }}{I_{\max }-I_{\min }} f(r)+I_{\min }
$$

where $f(r)$ is the function with respect to $r$ and $r$ is the Euclidean distance from point $f(r)$ to the iris center.

In our algorithm, accuracy of outer boundary localization depends on the distance function $f(r)$. Specifically, $f(r)$ enables smoothing of gradient patterns while keep the outer boundary sharp. To achieve this goal we let $f(r)=n r$.In our experiment we set $n=4$ for the database CASIA-IrisV3-Lamp released by Chinese Academy of Sciences.

To illustrate the gray-level transformation of the (5), we process an iris image as shown in Fig. 3 and Fig.4. . From the selected patch (as shown in Fig. 3) in the left or right of the pupil, it can be seen that from sclera to iris center there are some fluctuations in intensity caused by iris texture. Therefore if such fluctuations are flattened, accuracy of outer boundary localization will be improved (see in Fig.4). By gray-level transformation, the gradient of the iris texture area in the image is restrained, and the contrast of gradient between the iris and the sclera is enhanced.

\subsubsection{Iris Outer Boundary Localization}

For gray-level transformed image I_T, edge detection is performed with Canny detector [10]. Since there is the smoothing and edge connection in Canny detection, outer iris boundary can be extracted while most trivial responses will be eliminated by non-maximum supression [11].

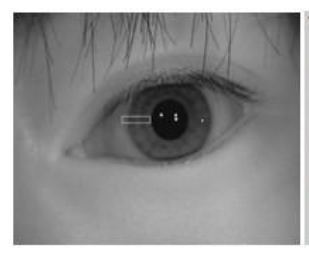

(1)

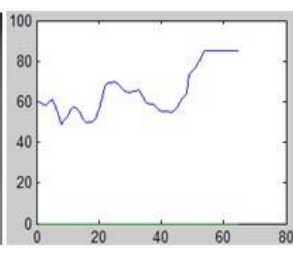

(2)

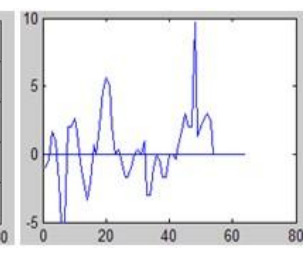

(3)
Fig. 3 The gray-level and gray-level gradient characteristics of the selected area (1) selected patch of original image (2) gray variation of the patch (3) gradient of the patch 


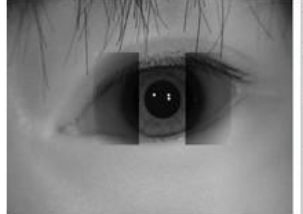

(1)

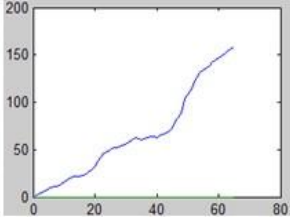

(2)

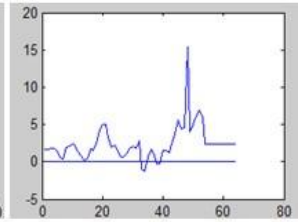

(3)
Fig. 4 The gray-level and gray-level gradient characteristics of the transformed area (1) transformed image (2) transformed area gray-level (3) gray-level gradient

The canny operator definition is defined as

$$
\left|\nabla G(x, y) * I_{-} T(x, y)\right|
$$

where

$$
\nabla \equiv\left(\frac{\partial}{\partial x}, \frac{\partial}{\partial y}\right)
$$

and

$$
|\nabla G(x, y)|=\frac{1}{2 \pi^{2}} \exp \left(-\frac{\left(x-x_{0}\right)^{2}+\left(y-y_{0}\right)}{2 \sigma^{2}}\right)
$$

In (6) and (8), $G(x, y)$ is a 2-D Gaussian function with the center $\left(x_{0}, y_{0}\right)$ and standard deviation $\sigma$. As the edge has been extracted in the transformed area in 1.2.1, and the trivial edges have been removed by threshold method, a set of edge points denoted as $\left(x_{j}, y_{j}\right) \mathrm{j}=1,2 \ldots, \mathrm{n}$ are obtained. Finally we detect $t$ the radius of outer boundary by improved Hough transform. The definition of the improved Hough transform is defined as

$$
H\left(x_{c}, y_{c}, r\right)=\sum_{j=1}^{n} h\left(x_{j}, y_{j}, x_{c}, y_{c}, r\right)
$$

where

$$
h\left(x_{j}, y_{j}, x_{c}, y_{c}, r\right)= \begin{cases}1, & \text { if } g\left(x_{j}, y_{j}, x_{c}, y_{c}, r\right)<10 \\ 0, & \text { otherwise }\end{cases}
$$

and

$$
g\left(x_{j}, y_{j}, x_{c}, y_{c}, r\right)=\left|\left(x_{j}-x_{c}\right)^{2}+\left(y_{j}-y_{c}\right)^{2}-r^{2}\right|
$$

$\left(x_{c}, y_{c}\right), r$ are the center and the radius of the outer boundary, respectively. This method uses the $g\left(x_{j}, y_{j}, x_{c}, y_{c}, r\right)<10$ instead of the original $g\left(x_{j}, y_{j}, x_{c}, y_{c}, r\right)=0$ in (10), and uses the $\left|\left(x_{j}-x_{c}\right)^{2}+\left(y_{j}-y_{c}\right)^{2}-r^{2}\right|$ instead of the original $\left(x_{j}-x_{c}\right)^{2}+\left(y_{j}-y_{c}\right)^{2}-r^{2}$ in (11). The parameter resetting improves the localization precision of the outer boundary. The group of $\left(x_{c}, y_{c}\right), r$ which make $H$ to be maximum is the parameter to determine outer boundary.

\section{Experimental Results}

In experiment we use the CASIA-IrisV3-Lamp database, provided by the Institute of Automation, Chinese Academy of Sciences, to evaluate our proposed method. The database contains 16440 iris images from 411 subjects, and there are 20 images per subject. Compared to CASIA-IrisV1 and CASIAIrisV2 databases, quality of images in CASIA-IrisV3-Lamp database is poor. Some images have bright spots in the pupil, and a considerable part of them have serious eyelashes, eyelid occlusion. Also in this database a great number of outer boundaries are ambiguous. For these reasons the Lamp database is difficult to deal with.

In our experiment three methods, Daugman's, Wildes' and level set [5], are implemented to the Lamp database. It is noted that before they are used, iris images firstly are decomposed by LP. We determine the localization accuracy subjectively. If the localization result of an image is close to the true boundaries in vision, it is regarded as accurate. The localization results are shown in Fig. 5, and the comparison of accuracy ratio is shown in table 1. It can be seen that our methods achieves a high performance than other three methods.

Our experiment is carried out in the PC of WinXP-SP3 OS, (Dual-core) CPU with $3.01 \mathrm{GHz}$, and 3GB Ram. The simulation software is Matlab7.6.

\section{Conclusion}

For non-ideal iris images there are often some shortcomings cause inaccurate iris localization. In this paper we propose the nonlinear gray-Level transformation method. Our method enhances the contrast between iris and sclera and reduces the gradient of the iris texture. The experimental result depict that our algorithm can overcome the aforemen-tioned difficukties effectively and improves the non-ideal iris localization accuracy compared with the classical algorithms.

\section{Acknowledgment}

This paper is supported by Scientific and Technological Development Program of Shandong Province (2011GGB1053), Ji'nan City University Independent Innovation Program (201202018, 201202001), Postdoctoral fund of Southeast University (1109000157).

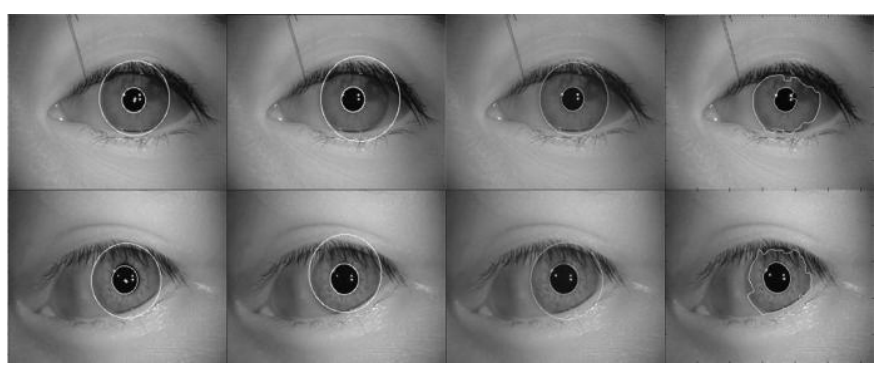
(1) Our method
(2) Daugman
(3) Wildes
(4) Level set

Fig. 5 The localization result

TABLE I The comparison results

\begin{tabular}{|c|c|}
\hline Method & Localization accuracy $(\%)$ \\
\hline The proposed method & 96.6 \\
\hline Daugman method & 82.2 \\
\hline Wildes method & 75.0 \\
\hline Level set & 31.4 \\
\hline
\end{tabular}




\section{References}

[1] Daugman J (1993). High confidence visual recognition of persons by a test of statistical independence. IEEE Trans. Pattern Analysis and Machine Intelligence, 15 (11):1148-1161.

[2] Daugman J (2007). New methods in iris recognition. IEEE Trans. System, Man, and Sybernetics-Part B: Cybernetics, 37 (5): 1167-1175.

[3] Wildes RP, Iris recognition: an emerging biometric technology. Proceedings of the IEEE, 1997, 85(9):348-1363.

[4] Wang, Y. H., Zhu Y., Tan T. N. (2002). Biometrics personal identification based on iris pattern , Acta Automatic Sinica, 28(1):1-10

[5] Roy K, Bhattacharya P, Suen CY (2011). Iris segmentation using variational level set method. Optics and Lasers in Engineering, 49 (4): 578-588.

[6] Pundlik S, Woodard D, Birchfield S (2010). Iris segmentation in nonideal images using graph cuts.Image Vision and Computing, 28 (12): 1671-1681.
[7] Miguel A. Luengo-Oroz, Emmanuel Faure, Jesús Angulo (2010). Robust iris segmentation on uncalibrated noisy images using mathematical morphology. Image Vision and Computing, 28 (2): 246-253.

[8] Wan, H. L., Han, M., Wang, T. (2012). Multiresolution Based Segmentation for Non-ideal Iris with Nonlinear Diffusion. Automation and Robotics, D. Yang, Editor. Springer Berlin Heidelberg: 107-111

[9] Ju, Y.W., Tian, Z., Zhang, Y. (2006). Efficient image segmentation method based on resolution and region information fusion. Chinese Optics Letters, 4(11): 639 642

[10] 10. Rafael C. Gonzalez, Richard E. Woods, Steven L. Eddins. (2009). Digital Image Processing(3rd edition).Beijing: Publishing House of Electronics Industry, 1-609

[11] Huang, J.L., Zheng, X.M. (2008). Improved image edge detection based on Canny operator. Computer and Applications, 44(25):170-172. 